\title{
Effects of gap junction intercellular communication on the docetaxel-induced cytotoxicity in rat hepatocytes
}

\author{
NAN TANG $^{1 *}$, JINGHUA LIU $^{1 *}$, BO CHEN ${ }^{1}$, YUAN ZHANG $^{2}$, \\ MEILING $\mathrm{YU}^{3}$, ZIQING CAI ${ }^{1}$ and HONGPENG CHEN ${ }^{4}$ \\ ${ }^{1}$ School of Pharmacy, Guangdong Medical University, Dongguan, Guangdong 523808; \\ ${ }^{2}$ The First Affiliated Hospital of Jinan University, Guangzhou, Guangdong 510632; ${ }^{3}$ Department of \\ Pharmacy, The First Affiliated Hospital of Bengbu Medical College, Bengbu, Anhui 233004; ${ }^{4}$ School of \\ Information Engineering, Guangdong Medical University, Dongguan, Guangdong 523808, P.R. China
}

Received December 16, 2015; Accepted January 24, 2017

DOI: $10.3892 / \mathrm{mmr} .2017 .6295$

\begin{abstract}
The effect of gap junction intercellular communication (GJIC) on docetaxel-induced hepatotoxicity and its underlying mechanisms are largely unknown. The present study involved investigating the effect of downregulating GJs derived from connexin (Cx) 32 in BRL-3A cells by three different mechanisms: Using a low-density culture; suppression of Cx32 using small interfering RNA; and use of the chemical inhibitor 2-aminoethoxydiphenyl borate (2-APB), all of which led to attenuated docetaxel hepatotoxicity. In order to investigate the relevant mechanisms involved, apoptosis and caspase activities of BRL-3A cells were determined. The increase of apoptosis and the activation of caspase- 3 and caspase- 9 , but not caspase-8, were detected following cell exposure with docetaxel, demonstrating that the mitochondrial-mediated apoptosis pathway is largely responsible for docetaxel hepatotoxicity. However, reduced apoptosis and caspase-3, and -9 activities were observed following docetaxel application when BRL-3A GJIC was deficient from the knockdown of Cx32 expression or pretreatment with 2-APB. These observations illustrate that GJs are important in docetaxel-induced hepatotoxicity. Furthermore, inhibition of GJIC could prevent amplification of toxicity to docetaxel. Due to GJIC blockage, this hepatoprotection was associated, in part, with decreasing apoptosis of BRL-3A cells through the mitochondrial pathway. The present study provides evidence for potential therapeutic targets for the treatment of docetaxel-induced liver injury.
\end{abstract}

Correspondence to: Dr Hongpeng Chen, School of Information Engineering, Guangdong Medical University, 1 Xincheng Road, Dongguan, Guangdong 523808, P.R. China

E-mail: chenhpgdmc@foxmail.com

*Contributed equally

Key words: docetaxel, gap junction, 2-APB, hepatotoxicity, connexin 32

\section{Introduction}

The liver is the primary organ involved in drug metabolism and disposition. Most drugs are excreted following biotransformation in the liver, in which some toxic metabolites, free radicals and reactive intermediates are produced, resulting in hepatic toxicity (1-3). As a result, the liver is a crucial target of drug toxicity.

Drug-induced liver injury (DILI) is a serious public health concern, often leading to a decline in drug efficacy, discontinuation of therapy, and even instigates liver failure and death $(4,5)$. These are the most significant reasons for withdrawal of drugs from the market (6-8). Therefore, novel strategies for the prevention and treatment of DILI are critical to future drug safety and efficacy.

Many types of drugs have reported DILI occurrences throughout treatment. Among them, antineoplastic agents are one of the most common types of drugs to cause DILI (9). Previous studies have demonstrated that most chemotherapeutic drugs or their metabolites may cause liver dysfunction and liver failure $(10,11)$. Docetaxel belongs to the groups of taxane antitumor agents, and has been widely used in the treatment of various forms of cancer alone or combination with other chemotherapeutic drugs (12-15). However, docetaxel-induced liver injury has, thus far, limited its clinical success $(16,17)$. Therefore, methods of reducing the hepatotoxicity of docetaxel are required.

Gap junctions (GJs) are plasma membrane channels composed of connexin (Cx). Six Cx monomers are assembled into a hemichannel (connexon), which docks to a counterpart from the adjacent cell to form a GJ channel. Small molecules, metabolites and second messengers can spread and transfer between neighboring cells via GJs. Gap junction intercellular communication (GJIC) is important in cell proliferation, differentiation and homeostasis $(18,19)$.

Recent studies have demonstrated that the hepatotoxicities of some drugs are associated with GJ function. Blocking GJ channels composed of $\mathrm{Cx} 32$ (a major $\mathrm{Cx}$ isoform in hepatocytes) can resist liver damage caused by acetaminophen in vitro and in vivo $(20,21)$. The protective effect may be linked to preventing the intercellular transmission of an 'injury signal' 
(such as free radicals) via GJs to adjacent cells. This action has been reported in D-galactosamine and carbon tetrachloride, where loss of GJIC reduced their hepatotoxicities (22). These evidences illustrate that GJs may become an important mediator for the treatment of DILI. However, there is little investigation into the action of GJs on docetaxel-induced liver injury. The purpose of this study was to determine the effect and possible mechanisms that underlie Cx32 GJ activity in docetaxel hepatotoxicity.

\section{Materials and methods}

Materials. Docetaxel was purchased from the National Institutes for Food and Drug Control (Beijing, China). 2-aminoethoxydiphenyl borate (2-APB), Hoechst 33258 , all primary and secondary antibodies for western blot analysis were obtained from Sigma-Aldrich; Merck-Millipore (Darmstadt, Germany). Lipofectamine ${ }^{\mathrm{TM}}$ 2,000, calcein-AM and cell culture reagents were purchased from Thermo Fisher Scientific, Inc. (Waltham, MA, USA). Cell Counting Kit-8 (CCK-8) was obtained from Dojindo Molecular Technologies, Inc. (Kumamoto, Japan). Caspase colorimetric activity assay kits were purchased from Beyotime Institute of Biotechnology (Haimen, China).

Cell culture. The rat liver-derived BRL-3A cell line used in this study was obtained from American Type Culture Collection (Manassas, VA, USA). Cells were cultured in Dulbecco's modified Eagle's medium supplemented with $10 \%$ fetal bovine serum (Gibco; Thermo Fisher Scientific, Inc.) and 1\% penicillin-streptomycin at $37^{\circ} \mathrm{C}$ in a $5 \% \mathrm{CO}_{2}$ incubator (Thermo Fisher Scientific, Inc.).

Drug treatment. Docetaxel and 2-APB were dissolved in dimethylsulfoxide (DMSO) and diluted in culture medium, in which the final concentration of DMSO was less than $0.1 \%$ (v/v). 2-APB was added to the cells at $50 \mu \mathrm{M}$ for $1 \mathrm{~h}$ prior to incubation with docetaxel and remained during the docetaxel treatment.

RNA interference. Cells were seeded at a density of 5,000 cells $/ \mathrm{cm}^{2}$ into plates and achieved $30-50 \%$ confluency by the time of Cx32 small interfering (si)RNA transfection. The negative control siRNA (NCsiRNA) or targeted Cx32 siRNA (Guangzhou Ribobio Co., Ltd., Guangzhou, China) were transfected into BRL-3A cells using Lipofectamine ${ }^{\mathrm{TM}}$ 2000. The synthetic sequences of siRNA for targeting Cx32 (si-Cx32) were as follows: si-Cx32-1, 5'-CACCAACAACACATAGAA A-3'; and si-Cx32-2, 5'-GCATCTGCATTATCCTCAA-3'. Knockdown of $\mathrm{Cx} 32$ expression and inhibition of GJIC were confirmed by western blot and parachute assays.

Assay of 'parachute' dye-coupling. The GJ function was determined by the 'parachute' dye-coupling assay as described previously $(23,24)$. Donor cells were labeled with $5 \mu \mathrm{M}$ calcein-AM, which is transformed intracellularly into calcein that exhibits GJ permeability. The donor cells were seeded on receiver cells at a 1:150 ratio (donor/receiver). The cells were cultured for $4 \mathrm{~h}$ at $37^{\circ} \mathrm{C}$ to form GJs, and then were monitored with a fluorescence microscope (IX71; Olympus Corporation,
Tokyo, Japan). For each condition, 12 different visual fields were assessed in triplicate. To evaluate the GJ function, the average number of receiver cells containing calcein dye/donor cell were counted and normalized against the vehicle control, containing no siRNA.

Western blot analysis. Western blot analysis was performed as described previously (25). Following sonication and centrifugation at $14,167 \mathrm{x} \mathrm{g}$ for $30 \mathrm{~min}$ at $4^{\circ} \mathrm{C}, 20 \mu \mathrm{g}$ whole-cell lysate was loaded per well, fractionated by $10 \%$ SDS-PAGE, and transferred onto a nitrocellulose membrane. The membranes were blocked with $5 \%$ milk for $1 \mathrm{~h}$ at room temperature. The antibodies against Cx32 (catalog no. C6344; 1:1,000) and $\beta$-actin, which was used as a loading control (catalog no. A2228; $1: 2,000)$ were applied overnight at $4^{\circ} \mathrm{C}$. The blots were then incubated with a horseradish peroxidase-conjugated goat anti-mouse IgG secondary antibody (catalog no. A4416) at a 1:4,000 dilution for $1 \mathrm{~h}$ at room temperature. Immunopositive bands were detected by an Amersham ECL $^{\text {Tм }}$ Plus Western Blotting Detection kit (GE Healthcare Bio-Sciences, Pittsburgh, PA, USA). The bands intensities were quantified by the Quantity One software version 4.6.2 (Bio-Rad Laboratories, Inc., Hercules, CA, USA).

Assay of cell viability. Cell viability was evaluated using the CCK-8 kit. Briefly, BRL-3A cells were exposed to docetaxel for $24 \mathrm{~h}$ followed by treatment with CCK-8 for another $3 \mathrm{~h}$ at $37^{\circ} \mathrm{C}$. The absorbance of each well was detected at $450 \mathrm{~nm}$ by a microplate reader (BioTek Instruments, Inc., Winooski, VT, USA). Four independent experiments were performed. The cell viability was assessed by normalizing the surviving fraction of the drug-treated group to the vehicle control.

Hoechst 33258 staining. BRL-3A cells were plated in 12-well dishes and cultured until they reached $80-100 \%$ confluency. The cells were exposed to agents, followed by washing with PBS and incubation with serum-free culture medium for another $24 \mathrm{~h}$. Cells were rinsed with PBS and fixed in $4 \%$ paraformaldehyde solution for $20 \mathrm{~min}$. Hoechst $33258(10 \mu \mathrm{g} / \mathrm{ml})$ was applied for $5 \mathrm{~min}$ in the dark to stain the nuclei of the cells. Apoptotic cells exhibited nucleus shrinkage with chromatin condensation. Following three washes with PBS, the cells were visualized and photographed by an Olympus IX71 fluorescence microscope. The cell apoptosis was assessed using the percentage of apoptotic cells/total cells under five randomly selected fields.

Caspase activities measurement. BRL-3A cells at 70-80\% confluency were incubated with docetaxel for $24 \mathrm{~h}$. Cells were harvested, lysed and centrifuged at $16,000 \times \mathrm{g}$ for $15 \mathrm{~min}$ at $4^{\circ} \mathrm{C}$. The supernatant of each sample was collected for caspase analysis. Caspase colorimetric assay kits evaluated activities of caspases-3, -8 and -9 . Absorbance detection was measured at $405 \mathrm{~nm}$ using a microplate reader (BioTek Instruments, Inc.).

Statistical analysis. Data were analyzed by the Sigma Plot software version 10.0 (Jandel Scientific, San Rafael, CA, USA) and expressed as the mean \pm standard error of the mean. The unpaired Student's t-test was used. $\mathrm{P}<0.05$ was considered to indicate statistical significance. 


\section{Results}

Effect of cell density on docetaxel-induced hepatotoxicity. For initially determining the effect of GJs on docetaxel hepatotoxicity, BRL-3A cells were cultured under low- and high-density conditions. GJ channels did not form as the cells were dispersed into single cells at low-density culture, while GJ formation was permitted at high-density culture for the cells that could contact each other (data not shown). Following exposure to docetaxel for $24 \mathrm{~h}$ in the two density conditions, cell survival was evaluated by CCK-8 assay. As demonstrated in Fig. 1A, docetaxel reduced cell survival in a concentration-dependent manner in the cases of low- and high-density. However, the survival fractions in low-density (GJ absence) were higher than that of cells in high-density (GJ presence) at concentrations of docetaxel up to $20 \mu \mathrm{M}$. In addition, a significant difference of cell viability was observed at 10 and $20 \mu \mathrm{M}(\mathrm{P}<0.05)$. The increased ratios of the survival fractions between low- and high-density conditions were more than $30.0 \%$ at docetaxel concentrations of 10 and $20 \mu \mathrm{M}(\mathrm{P}<0.05$; Fig. 1B). These results indicate that docetaxel hepatotoxicity is dependent on cell density and the toxic effect is decreased in blocking intercellular communication.

Inhibition ofGJICreduces the docetaxel-inducedhepatotoxicity. Since the reduced toxicity of docetaxel was attributed to the low-density culture, the next step involved further investigating the role of GJIC on docetaxel hepatotoxicity. Two methods were applied to regulate the GJs composed of Cx32 (Cx32 GJ) in BRL-3A cells: i) knockdown Cx32 expression by siRNA and ii) using the chemical inhibitor 2-APB (26). The expression of Cx32 was confirmed by western blot analysis and was markedly downregulated by transfection with si-Cx32-1 relative to the vehicle control and NCsiRNA (Fig. 2A). The GJIC inhibition of si-Cx32-1 (Fig. 2B) and 2-APB (Fig. 2C), were assessed by 'parachute' dye-coupling assay.

In the high-density group, the survival of Cx32-knockdown cells was significantly increased compared with the negative control treated cells in the presence of $10 \mu \mathrm{M}$ docetaxel, by a factor of $1.4(\mathrm{P}<0.05$; Fig. 3A). Treatment of BRL-3A cells with $50 \mu \mathrm{M} 2$-APB under high density conditions increased the cell viability from 57.3 to $75.5 \%$ during treatment with $10 \mu \mathrm{M}$ docetaxel $(\mathrm{P}<0.05$; Fig. 3B). These results demonstrated that inhibition of $\mathrm{GJ}$ function by either $\mathrm{Cx} 32$-knockdown or chemical inhibitor significantly reduces the hepatotoxicity of docetaxel.

Influence of GJ on docetaxel-induced apoptosis. To illustrate whether apoptosis is involved in the protective effect against docetaxel cytotoxicity by blocking GJs, Hoechst 33258 staining was used to evaluate the apoptosis rates of BRL-3A cells with or without GJIC. As demonstrated in Fig. 4A, the cell nuclei showed uniformly blue and smooth edges when cells were incubated with vehicle control. However, following treatment with docetaxel, some cells exhibited typical apoptosis characteristics, such as nuclei shrinkage and fragmentation leading to strong blue fluorescence. The apoptosis rates in BRL-3A cells pretreated with siRNA or 2-APB prior to docetaxel treatment was significantly decreased, by 55.0 and $50.4 \%$, respectively, compared with docetaxel only $(\mathrm{P}<0.05$; Fig. $4 \mathrm{~B})$. These observations demonstrated that reduced apoptosis is

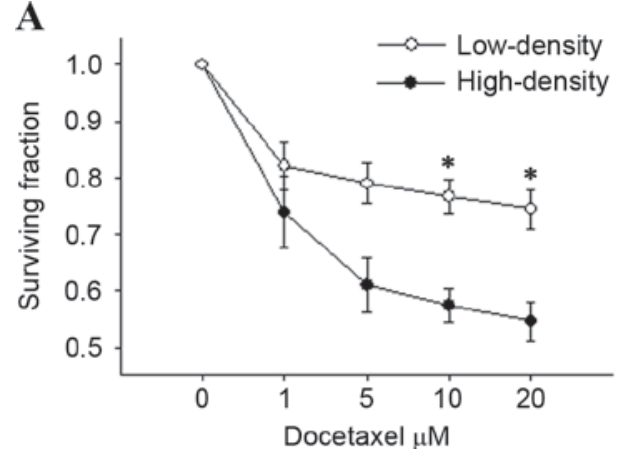

B

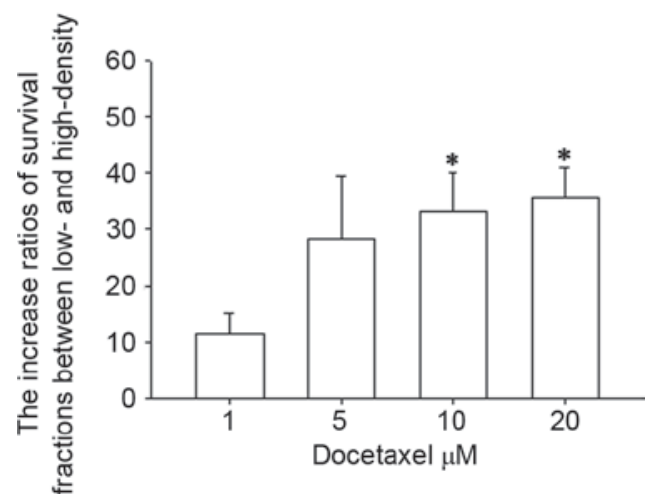

Figure 1. Effect of cell density on the hepatotoxicity induced by docetaxel in BRL-3A cells. (A) Cell survival was measured by CCK-8 assay following exposure to docetaxel at a range of concentrations for $24 \mathrm{~h}$ at high- and low-cell densities. Data are expressed as the mean \pm standard error of the mean from four repeats. ${ }^{*} \mathrm{P}<0.05$ vs. high-density group. (B) The ratios of cell survival between treatments in low- and high-density. Data are expressed as the mean \pm standard error of the mean. ${ }^{*} \mathrm{P}<0.05$ vs. $1 \mu \mathrm{M}$ docetaxel.

largely responsible for the protective effect of GJ inhibition on docetaxel hepatotoxicity.

Effects of GJ on caspase-3, -8, -9 activities. The caspase cascade system serves a vital role in the process of apoptosis. To investigate the possible apoptotic pathways, the activities of caspase-3, -8 and -9 in BRL-3A cells exposed to $10 \mu \mathrm{M}$ docetaxel were examined in the presence or absence of GJIC. As presented in Fig. 5, caspase-3 and caspase-9, but not caspase-8, were activated following treatment of cells with $10 \mu \mathrm{M}$ docetaxel for $24 \mathrm{~h}(\mathrm{P}<0.05$, control vs. docetaxel). However, knockdown of Cx32 expression and 2-APB treatment both significantly reduced the increased caspase- 3 and caspase- 9 activities, compared with docetaxel treatment only ( $\mathrm{P}<0.05$; Fig. 5), while having no effect on caspase- 8 activity $(\mathrm{P}>0.05$; Fig. 5). These observations indicate that docetaxel-induced cytotoxicity may be associated with caspase- 3 and caspase- 9 activations, and is attenuated through this apoptosis pathway by blocking GJIC.

\section{Discussion}

The present study illustrates that the toxicity of docetaxel is mediated by GJIC in BRL-3A cells. GJIC was downregulated using 3 methods: Low-density culture, knockdown of Cx32 expression through siRNA transfection, and application of the GJ chemical inhibitor, 2-APB. All 3 methods led to reduced docetaxel cytotoxicity, which reduced the toxic effect to 
A
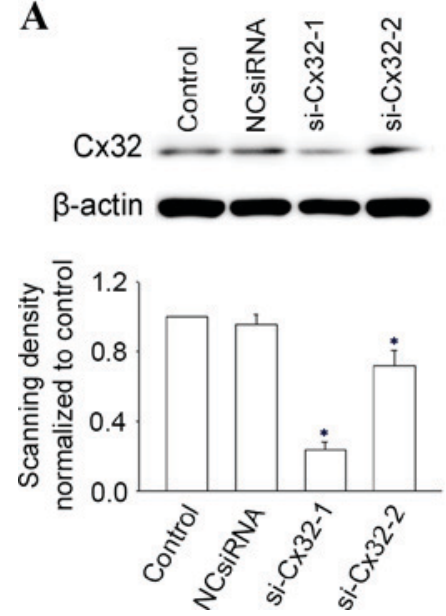

B

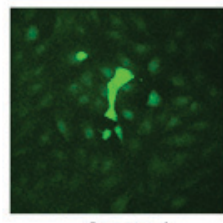

Control

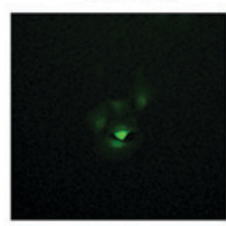

si-Cx32-1

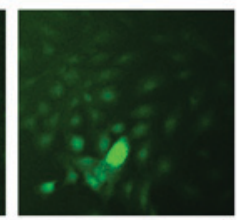

NCsiRNA

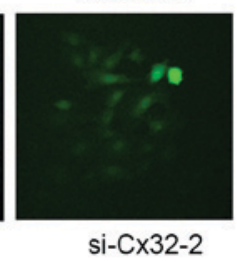

C

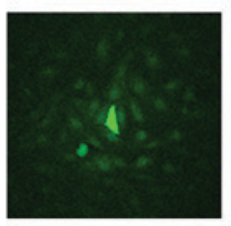

Control

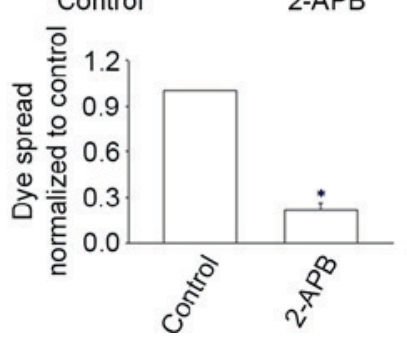

Figure 2. Inhibition of GJIC by siRNA and 2-APB. (A) Western blot analysis was used to evaluate Cx32 expression following Cx32-siRNA transfection in BRL-3A cells. The results obtained from densitometry of the blots are given as the mean \pm standard error of the mean (three repeats). "P<0.05 vs. control. (B) Fluorescence images of dye spread in BRL-3A cells transfected Cx32-siRNA by parachute dye-coupling assay. Cells in which Cx32 was knocked-down by siRNA exhibited lower dye spread when compared with the vehicle control (no siRNA) and NCsiRNA. (C) 2-APB inhibited dye transfer in BRL-3A cells, as demonstrated by the parachute dye-coupling assay. "P<0.05 vs. Control. GJIC, gap junction intercellular communication; siRNA, small interfering RNA; 2-APB, 2-aminoethoxydiphenyl borate; $\mathrm{Cx}$, connexin; $\mathrm{NC}$, negative control.
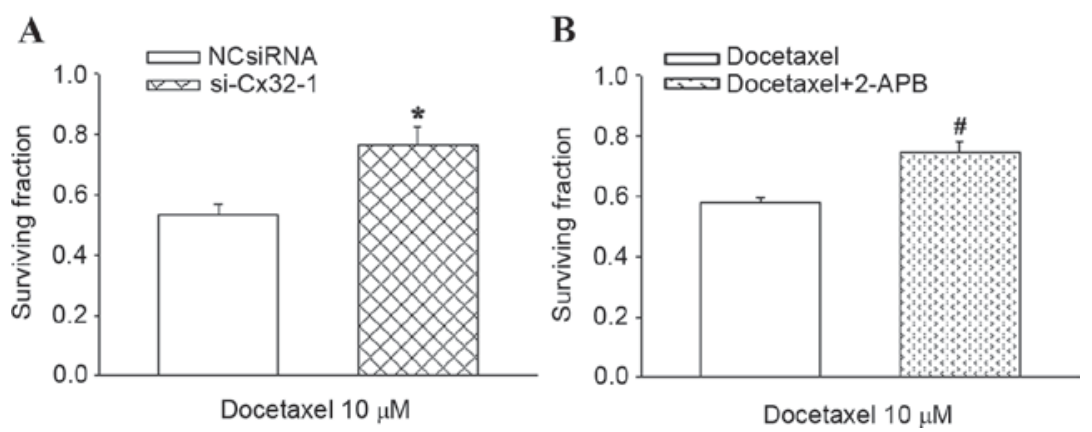

Figure 3. Hepatotoxicity of docetaxel in BRL-3A cells is reduced when gap junction intercellular communication is blocked by Cx32-knockdown and pretreatment 2-APB. (A) Cell survival was measured by CCK-8 assay following transfection with NCsiRNA or si-Cx32-1, then $24 \mathrm{~h}$ treatment with $10 \mu \mathrm{M}$ docetaxel at high cell density. (B) Cell survival was measured by CCK- 8 assay following treatment with $10 \mu \mathrm{M}$ docetaxel $+/-50 \mu \mathrm{M} 2$-APB at high cell density. Data are presented as the mean \pm standard error of the mean from four repeats. "P $<0.05$ vs. NCsiRNA, ${ }^{\text {} P}<0.05$ vs. docetaxel. Cx, connexin; 2-APB, 2-aminoethoxydiphenyl borate; CCK-8, Cell Counting Kit-8; NC, negative control; siRNA, small interfering RNA.

BRL-3A cells. To the best of our knowledge, the present findings revealed for the first time that inhibition GJIC exerts a protective effect on liver injury caused by docetaxel.

Apoptosis is an orderly cell death program and is critical for the maintenance of cell homeostasis, which is one of the main mechanisms in antineoplastic agents' cytotoxicity (27-29). The caspase cascade system exerts an important role in initiating and amplifying apoptotic signals. There are 2 major pathways resulting in caspase activation: One is the mitochondrial pathway, mainly mediated by the caspase- 9 ; the other is death receptor pathway, mainly mediated by the caspase- 8 (30). The two pathways both go on to activate caspase-3, thereby causing the morphological and biochemical changes (31). In the current study, docetaxel-induced apoptosis was demonstrated to be related to its hepatotoxicity. Furthermore, docetaxel increases caspase-9, rather than caspase-8, to activate downstream caspase-3, indicating that mitochondrial pathway is largely responsible for the docetaxel hepatotoxicity. While this hepatotoxicity was attenuated when blocking GJs activities mainly via decreasing apoptosis, for caspase-3 (the important executive factor of apoptosis) and the upstream factor of caspase-9, not caspase-8, were influenced. Results of the current study demonstrated that GJIC regulated the biochemical factors induced by docetaxel through the mitochondrial pathway but not the death receptor pathway.

GJ channels composed of different $\mathrm{Cx}$ exhibit distinct permeability for signal molecules. For instance, adenosine permeates Cx32 channels approximately 12 times more effectively than $\mathrm{Cx} 43$ channel; the permeability of inositol 1,4,5-trisphosphate $\left(\mathrm{IP}_{3}\right)$ through $\mathrm{Cx} 32$ channels is higher than that of Cx26 channels (32). The present results indicated that docetaxel hepatotoxicity was reduced when Cx32 GJ function was suppressed, suggesting some 'injury signals' induced by docetaxel were prevented transmission through Cx32 GJs. Free radicals and parent drugs are likely candidates. Previous studies have reported that oxidative stress is a widely accepted consequence of hepatotoxin exposure and has a close relationship with mitochondrial function $(33,34)$. Free radicals as the oxidative stress signals can propagate through Cx32 GJs and thus amplify this injury (20). Docetaxel may produce a direct toxic effect, causing mitochondrial damage, which may pass through Cx32 GJs by passive transference due to its molecular 
$\mathbf{A}$

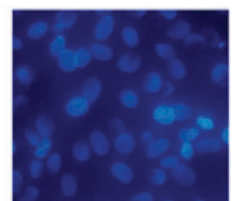

Control

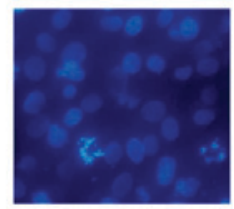

Docetaxel/si-Cx32-1

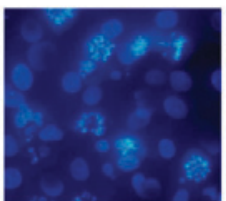

Docetaxel

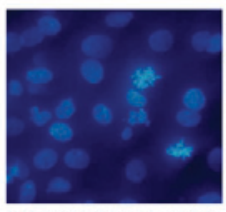

Docetaxel/2-APB

\section{B}

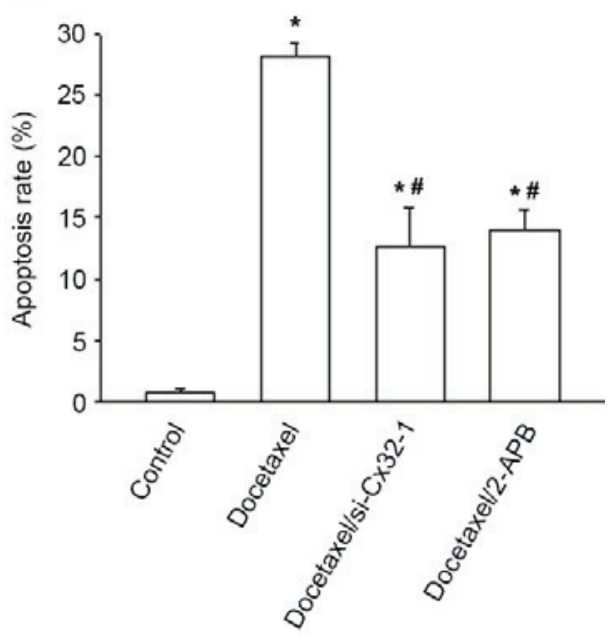

Figure 4. Effects of siRNA-mediated Cx32 knockdown and 2-APB on docetaxel-induced apoptosis. Apoptosis of BRL-3A cells was determined by Hoechst 33258 staining following transfection with si-Cx32-1 or treatment with $50 \mu \mathrm{M}$ 2-APB, followed by exposure to $10 \mu \mathrm{M}$ docetaxel. (A) Fluorescence imaging of BRL-3A cells stained with Hoechst 33258 (original magnification, x200). (B) Apoptosis rate of BRL-3A cells in the presence or absence of gap junction intercellular communication. The results are expressed as the mean \pm standard error of the mean. ${ }^{*} \mathrm{P}<0.05$ vs. vehicle control, ${ }^{\#} \mathrm{P}<0.05$, vs. docetaxel. siRNA, small interfering RNA; Cx, connexin; 2-APB, 2-aminoethoxydiphenyl borate; si(RNA), small interfering (RNA).
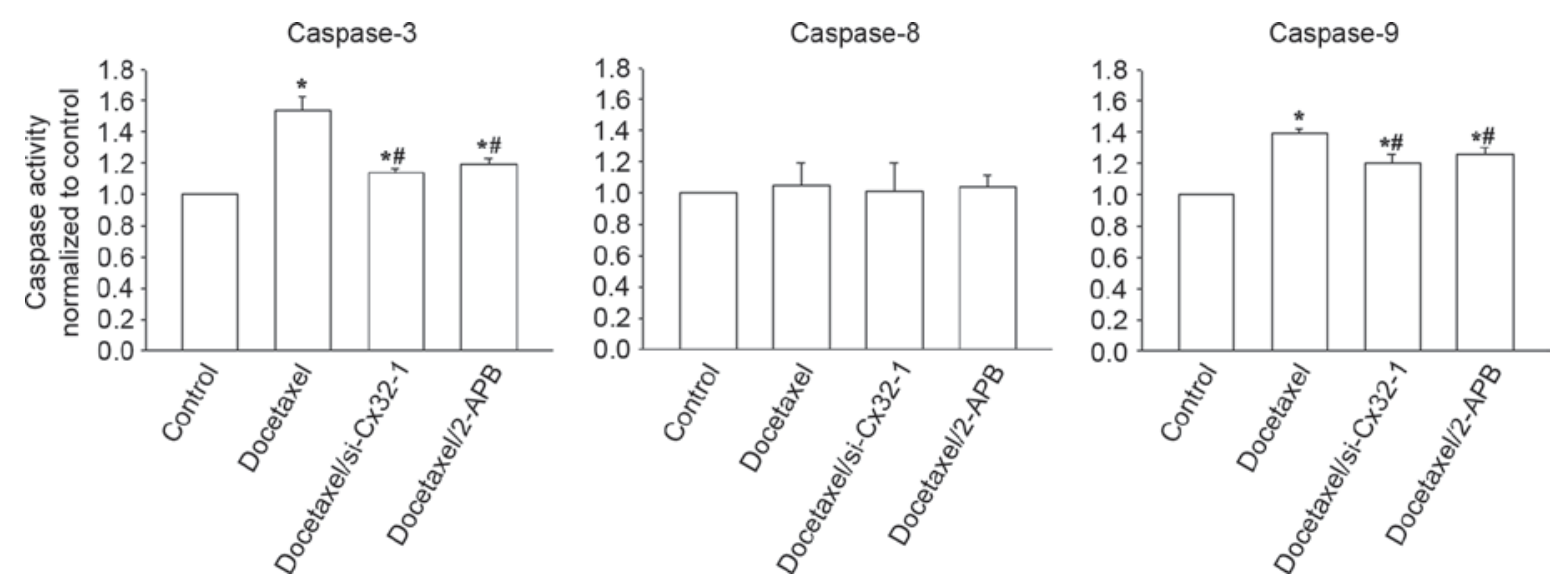

Figure 5. Effects of GJIC on caspase-3, -8, -9 activities in docetaxel-treated BRL-3A cells. GJIC was downregulated by siRNA-mediated Cx32 knockdown and treatment with $50 \mu \mathrm{M}$ 2-APB. The caspase activities were measured by colorimetric assay kits following exposure of BRL-3A cells to $10 \mu \mathrm{M}$ docetaxel for $24 \mathrm{~h}$. Data are expressed as the mean \pm standard error of the mean. $\mathrm{P}<0.05$ vs. vehicle control, ${ }^{~} \mathrm{P}<0.05$ vs. docetaxel. GJIC, gap junction intercellular communication; si(RNA), small interfering (RNA); Cx, connexin; 2-APB, 2-aminoethoxydiphenyl borate.

weight $(807.9 \mathrm{kDa})$, which is less than the limit of GJ permeable molecules. Nevertheless, the properties of 'injury signals' and their underlying mechanisms have yet to be explored further.
A previous study demonstrated that the cytotoxicity of docetaxel was enhanced at presence of GJIC in Cx32-transfected HeLa cells (23). Therefore, the therapeutic 
efficacy and hepatotoxicity of docetaxel are likely to be affected by GJ function. For lack of GJIC in numerous cancers $(35,36)$, inhibition of GJs in liver cells may be a promising strategy for the treatment of docetaxel-induced hepatotoxicity. However, in some forms of carcinoma with GJIC retention $(37,38)$, the impact on docetaxel efficacy needs to be considered when GJs are used as the target for the treatment of hepatic injury.

In summary, the results of the present study demonstrate that downregulation of GJs derived from Cx32 could elicit a protective role against docetaxel-induced hepatotoxicity, which is mediated by GJIC. In addition, this hepatoprotection appears to be due to reduced caspase-3, -9 activation, thereby decreasing the apoptosis and cell toxicity of docetaxel. Further studies are required to examine the effects of GJ on the docetaxel-induced cytotoxicity in other hepatocyte strains and in vivo.

\section{Acknowledgements}

The present study was supported by the grants of the National Natural Science Foundation of China (grant no. 81400619), the Guangdong Province Public Interest Research and Capacity Building Special Fund (grant no. 2014A020212508) and the Science Foundation for the Doctoral Program of Guangdong Medical University (grant no. B2013011).

\section{References}

1. Corsini A and Bortolini M: Drug-induced liver injury: The role of drug metabolism and transport. J Clin Pharmacol 53: 463-474, 2013.

2. Amacher DE: The primary role of hepatic metabolism in idiosyncratic drug-induced liver injury. Expert Opin Drug Metab Toxicol 8: 335-347, 2012

3. Claesson A and Spjuth O: On mechanisms of reactive metabolite formation from drugs. Mini Rev Med Chem 13: 720-729, 2013.

4. Daly AK: Drug-induced liver injury: Past, present and future. Pharmacogenomics 11: 607-611, 2010

5. Hussaini SH and Farrington EA: Idiosyncratic drug-induced liver injury: An overview. Expert Opin Drug Saf 6: 673-684, 2007.

6. Kaplowitz N: Idiosyncratic drug hepatotoxicity. Nat Rev Drug Discov 4: 489-499, 2005.

7. Senior JR: Evolution of the food and drug administration approach to liver safety assessment for new drugs: Current status and challenges. Drug Saf 37 (Suppl 1): S9-S17, 2014.

8. Regev A: Drug-induced liver injury and drug development: Industry perspective. Semin Liver Dis 34: 227-239, 2014.

9. Meier Y, Cavallaro M, Roos M, Pauli-Magnus C, Folkers G, Meier PJ and Fattinger K: Incidence of drug-induced liver injury in medical inpatients. Eur J Clin Pharmacol 61: 135-143, 2005.

10. Thatishetty AV, AgrestiN and O'BrienCB: Chemotherapy-induced hepatotoxicity. Clin Liver Dis 17: 671-686, ix-x, 2013.

11. Bahirwani R and Reddy KR: Drug-induced liver injury due to cancer chemotherapeutic agents. Semin Liver Dis 34: 162-171, 2014.

12. Kim HS, Ryu MH, Zang DY, Ryoo BY, Yang DH, Cho JW, Lim MS, Kim MJ, Han B, Choi DR, et al: Phase II study of docetaxel, oxaliplatin, and S-1 therapy in patients with metastatic gastric cancer. Gastric Cancer 19: 579-585, 2016.

13. Gelmon K: The taxoids: Paclitaxel and docetaxel. Lancet 344 : 1267-1272, 1994

14. Pazdur R, Kudelka AP, Kavanagh JJ, Cohen PR and Raber MN: The taxoids: Paclitaxel (Taxol) and docetaxel (Taxotere). Cancer Treat Rev 19: 351-386, 1993.

15. Dancey J, Shepherd FA, Gralla RJ and Kim YS: Quality of life assessment of second-line docetaxel versus best supportive care in patients with non-small-cell lung cancer previously treated with platinum-based chemotherapy: Results of a prospective, randomized phase III trial. Lung Cancer 43: 183-194, 2004.
16. Wang Z, Liang X, Yu J, Zheng X, Zhu Y, Yan Y, Dong N, Di L, Song G, Zhou X, et al: Non-genetic risk factors and predicting efficacy for docetaxel-drug-induced liver injury among metastatic breast cancer patients. J Gastroenterol Hepatol 27: 1348-1352, 2012.

17. Liang X, Zhang J, Zhu Y, Lu Y, Zhou X, Wang Z, Yu J, Yan Y, Di L, Che L, et al: Specific genetic polymorphisms of IL10-592 AA and IL10-819 TT genotypes lead to the key role for inducing docetaxel-induced liver injury in breast cancer patients. Clin Transl Oncol 15: 331-334, 2013.

18. Maeda $S$ and Tsukihara T: Structure of the gap junction channel and its implications for its biological functions. Cell Mol Life Sci 68: 1115-1129, 2011

19. Harris AL: Connexin channel permeability to cytoplasmic molecules. Prog Biophys Mol Biol 94: 120-143, 2007.

20. Patel SJ, Milwid JM, King KR, Bohr S, Iracheta-Vellve A, Li M, Vitalo A, Parekkadan B, Jindal R and Yarmush ML: Gap junction inhibition prevents drug-induced liver toxicity and fulminant hepatic failure. Nat Biotechnol 30: 179-183, 2012.

21. Naiki-Ito A, Asamoto M, Naiki T, Ogawa K, Takahashi S, Sato S and Shirai T: Gap junction dysfunction reduces acetaminophen hepatotoxicity with impact on apoptotic signaling and connexin 43 protein induction in rat. Toxicol Pathol 38: 280-286, 2010.

22. Asamoto M,Hokaiwado N, Murasaki T and Shirai T: Connexin 32 dominant-negative mutant transgenic rats are resistant to hepatic damage by chemicals. Hepatology 40: 205-210, 2004.

23. Tang N, Wang Q, Wu D, Zhang S, Zhang Y and Tao L: Differential effects of paclitaxel and docetaxel on gap junctions affects their cytotoxicities in transfected HeLa cells. Mol Med Rep 8: 638-644, 2013.

24. Goldberg GS, Bechberger JF and Naus CC: A pre-loading method of evaluating gap junctional communication by fluorescent dye transfer. Biotechniques 18: 490-497, 1995.

25. Hong X, Wang Q, Yang Y, Zheng S, Tong X, Zhang S, Tao L and Harris AL: Gap junctions propagate opposite effects in normal and tumor testicular cells in response to cisplatin. Cancer Lett 317: 165-171, 2012.

26. Tao L and Harris AL: 2-aminoethoxydiphenyl borate directly inhibits channels composed of connexin26 and/or connexin32. Mol Pharmacol 71: 570-579, 2007.

27. Todd RC and Lippard SJ: Inhibition of transcription by platinum antitumor compounds. Metallomics 1: 280-291, 2009.

28. Bhalla KN: Microtubule-targeted anticancer agents and apoptosis. Oncogene 22: 9075-9086, 2003.

29. Fulda S: Modulation of apoptosis by natural products for cancer therapy. Planta Med 76: 1075-1079, 2010.

30. Park HH, Lo YC, Lin SC, Wang L, Yang JK and Wu H: The death domain superfamily in intracellular signaling of apoptosis and inflammation. Annu Rev Immunol 25: 561-586, 2007.

31. Tong X, Dong S, Yu M, Wang Q and Tao L: Role of heteromeric gap junctions in the cytotoxicity of cisplatin. Toxicology 310 : 53-60, 2013.

32. Niessen H, Harz H, Bedner P, Krämer K and Willecke K: Selective permeability of different connexin channels to the second messenger inositol 1,4,5-trisphosphate. J Cell Sci 113: $1365-1372,2000$

33. Circu ML and Aw TY: Glutathione and apoptosis. Free Radic Res 42: 689-706, 2008.

34. Friesen C, Kiess Y and Debatin KM: A critical role of glutathione in determining apoptosis sensitivity and resistance in leukemia cells. Cell Death Differ 11 (Suppl 1): S73-S85, 2004.

35. Mesnil M, Crespin S, Avanzo JL and Zaidan-Dagli ML: Defective gap junctional intercellular communication in the carcinogenic process. Biochim Biophys Acta 1719: 125-145, 2005.

36. Loewenstein WR and Kanno Y: Intercellular communication and the control of tissue growth: Lack of communication between cancer cells. Nature 209: 1248-1249, 1966.

37. Hanna EA, Umhauer S, Roshong SL, Piechocki MP, Fernstrom MJ, Fanning JD and Ruch RJ: Gap junctional intercellular communication and connexin 43 expression in human ovarian surface epithelial cells and ovarian carcinomas in vivo and in vitro. Carcinogenesis 20: 1369-1373, 1999.

38. Zhang W, DeMattia JA, Song H and Couldwell WT: Communication between malignant glioma cells and vascular endothelial cells through gap junctions. J Neurosurg 98: 846-853, 2003. 